Didáctica Geográfica n 20, 2019, pp. 173-192

ISSN: 0210-492-X

D.L: M-3736-2014

\title{
DIVULGAR LA GEOGRAFÍA A PARTIR DE UN TRABAJO COLABORATIVO EN LA SEMANA DE LA CIENCIA, LA TECNOLOGÍA Y LA INNOVACIÓN. EL STAND “DEL DATO AL GRÁFICO CLIMÁTICO”
}

Orbange Ormaetxea Arenaza ${ }^{1}$; Alexander Maiz Trojaola²; Rubén Cuesta López²; David Otxoa Foronda ${ }^{2}$

Recibido: 17/05/2019

Aceptado 04/09/2018

\section{RESUMEN:}

En este trabajo se presenta una propuesta de divulgación científica realizada a través de un stand durante un lustro y en el marco de la Semana de la Ciencia, la Tecnología y la Innovación de la Universidad del País Vasco UPV/EHU. Una experiencia que ha tenido como fin mostrar a una sociedad diversa una parte de la ciencia geográfica y los fundamentos tecnológicos y paramétricos de los que necesita. Para ello se han diseñado diferentes actividades de carácter complementario cuyo desarrollo metodológico y resultados se exponen a continuación.

\section{Palabras Clave:}

Divulgación científica, stand, geografía, datos atmosféricos, trabajo colaborativo.

\footnotetext{
${ }^{1}$ Departamento de Geografía, Prehistoria y Arqueología. Universidad del País Vasco, UPV/ EHU. orbange.ormaetxea@ehu.eus

2 Departamento de Tecnología Electrónica. Universidad del País Vasco, UPV/EHU.
} 


\section{Abstract:}

This work presents the proposal of scientific dissemination carried out through a stand during a five-year period within the framework of the Science, Technology and Innovation Week of the University of the Basque Country UPV / EHU. An experience that has had to show to a diverse society a part of the geographical science and the technological and parametric basis of which it needs. To this end, different complementary activities have been designed whose methodological development and results are described below.

\section{KEYWORDS:}

Scientific divulgation, stand, geography, atmospheric data, collaborative work.

\section{RÉSUMÉ:}

Cet article présente une proposition de vulgarisation scientifique réalisée sur cinq années consécutives par le biais d'un stand dans le cadre de la Semaine de la Science, de la Technologie et de l'Innovation de l'Université du Pays Basque UPV / EHU. Une expérience qui a eu pour but de montrer à une société diversifiée un volet de la science géographique et des fondements technologiques et paramétriques dont elle a besoin. À cette fin, différentes activités complémentaires ont été conçues, dont le développement méthodologique et les résultats sont décrits ci-dessous.

\section{MotS-CLÉS:}

Divulgation scientifique, stand, géographie, données atmosphériques, travail collaboratif.

\section{INTRODUCCIÓN}

A pesar de que como señalan algunos autores (Fernández, Mecha y Milán, 2018, p.7) "la divulgación no ha aportado tradicionalmente nada positivo al currículo, ni ha sido demasiado reconocida por los colegas", los docentes e investigadores debemos ser activos también en la difusión de conocimiento. La propia universidad a la que pertenecemos quienes firmamos este artículo, la Universidad del País Vasco/Euskal Herriko Unibertsitatea, tiene como lema "Eman ta zabal zazu/Dalo y difúndelo". Según el Artículo 4.1 de sus Estatutos (Universidad del País Vasco/Euskal Herriko Unibertsitatea, 2011, p. 10-11), entre otros, son fines de la misma: "la creación, crítica y transmisión del saber, contribuyendo al avance del conocimiento y al desarrollo social mediante la investigación y la transferencia de sus resultados a la sociedad; la participación en el enriquecimiento del patrimonio intelectual, humanístico, cultural y científico de la sociedad vasca y en su desarrollo económico y bienestar social y, la difusión y 
proyección universitarias". Para cumplir con ese compromiso esta universidad asume desde hace tres lustros (Orruño, Bikandi, San Millán y Arana, 2015, p.36) y a través, inicialmente de su Vicerrectorado de Investigación y ahora, de su Vicerrectorado de Desarrollo Científico y Transferencia, la responsabilidad de la organización anual de actividades asociadas a la Semana de la Ciencia, la Tecnología y la Innovación (SCTI) en los tres Territorios Históricos de Euskadi.

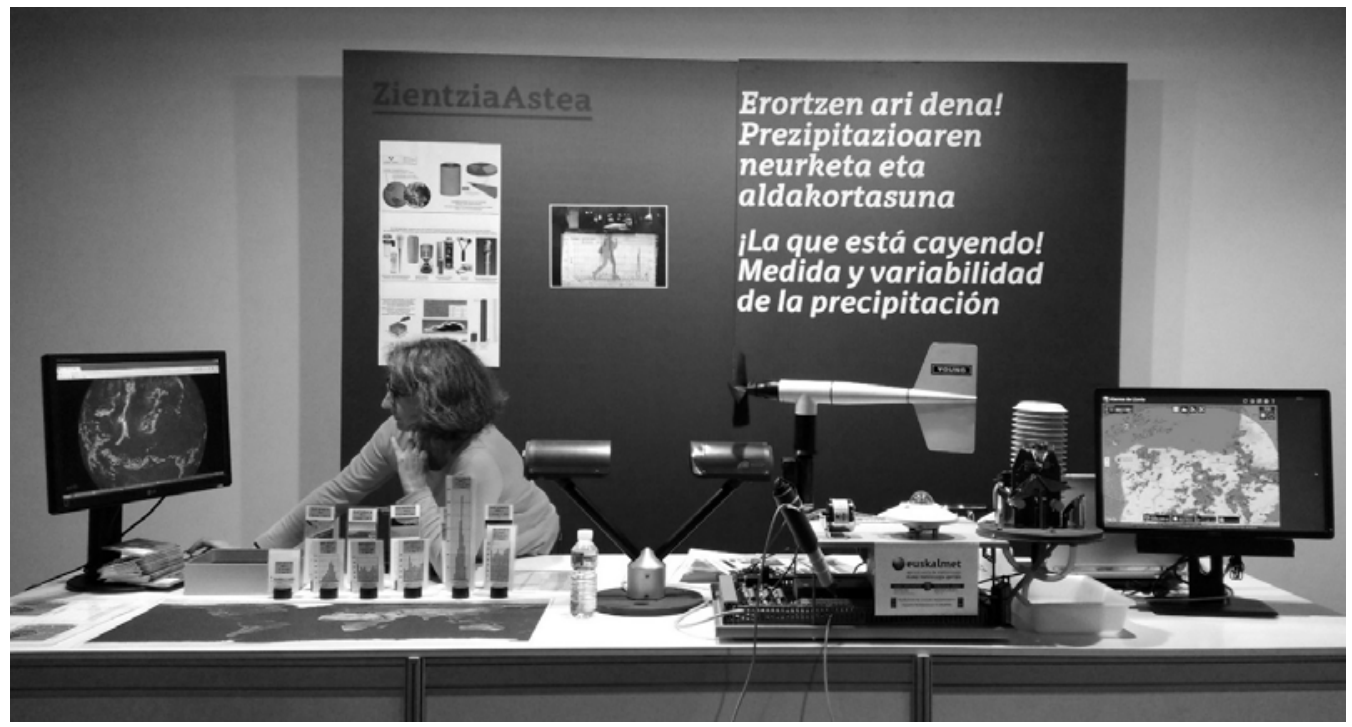

FiguRA $N^{\circ} 1$. Imagen general del stand de la Semana de la Ciencia, la Tecnología y la Innovación (SCTI) de 2014 “LLa que está cayendo! Medida y variabilidad de la precipitación”.

En este marco y desde 2009, varias profesoras del Departamento de Geografía, Prehistoria y Arqueología hemos llevado a cabo diversas propuestas de divulgación de la Geografía similares a las realizadas por otras universidades (Mecha, 2010) a través de un itinerario didáctico y un stand en Vitoria-Gasteiz (campus universitario de Álava) con diversos lemas: "Geografía" (2009), "Interpretar los paisajes: una perspectiva geográfica" (2010) y "Geografía: instrumentos y materiales" (2011-12). En 2014 y en colaboración con miembros del personal del Laboratorio de Calibración de Sensores Meteorológicos de la Escuela de Ingeniería de Vitoria-Gasteiz, diseñamos y desarrollamos una nueva actividad de stand que se ha realizado anualmente hasta 2018 y que tiene por lema "Del dato al gráfico climático" (el primer año el título fue ";La que está cayendo! Medida y variabilidad de la precipitación") (Figura $n^{\circ} 1$ ) y como objetivos los siguientes:

- dar acceso a información de carácter científico, en este caso relativo a variables climáticas, a un público general y diverso; 
- interactuar de una forma amena, a través de una actividad informal, con el fin de generar interés en la práctica tecnológica y geográfica de conocimiento e investigación;

- mostrar a los ciudadanos la necesidad y el valor de la colaboración en el trabajo científico.

\section{MÉTODO Y MATERIALES}

Para la consecución de esos objetivos se ha diseñado una batería de actividades teniendo como premisa la diversidad del público que va a visitar el stand. La SCTI se celebra en Vitoria-Gasteiz de jueves a domingo (noviembre) y, mientras en los dos primeros días es el alumnado de Educación Secundaria Obligatoria y Bachillerato quien acude al lugar donde se celebra el evento, el fin de semana está abierto al público general.

Las actividades diseñadas y desarrolladas consisten en:

- Exposición de instrumentos de medida de una estación meteorológica y presentación por parte de los colaboradores de las variables paramétricas y de las técnicas de medición.

- Muestra de los datos atmosféricos tratados para su utilización en Geografía: análisis de procesos y caracterización climática.

- Adaptándose al público que en cada momento se encuentra ante el stand los colaboradores realizan las actividades con diversas estrategias de presentación y desarrollo (Tablas $\mathrm{n}^{\circ} 1 \mathrm{y} \mathrm{n}^{\circ}$ 2) y siempre, tras finalizar la exposición de la Actividad A y para enlazarlo con la Actividad B atendiendo al segundo objetivo, se indica: "Pues con todos estos datos una vez verificados, en Geografía se utilizan para...".

El método de transmisión de la información en este apartado y para el público juvenil y adulto es de tipo deductivo. Desde lo más general, distinguiendo entre tiempo meteorológico y clima, meteorología y climatología, dinámica atmosférica, etc..., hasta los hechos más precisos recogidos en datos, gráficos y cartografía (Ramos y Calonge, 2014) incluyendo el recurso a la escala del territorio más cercano (datos medios anuales y gráficos de la Comunidad Autónoma del País Vasco y datos diarios de la ciudad de Vitoria-Gasteiz). 
Divulgar la Geografía a partir de un trabajo colaborativo en la Semana de la Ciencia,...

\begin{tabular}{|l|c|l|}
\hline Actividad & Público & \multicolumn{1}{c|}{ Preguntas de introducción a la actividad } \\
\hline \multirow{2}{*}{} & & (Para todos los instrumentos): \\
\cline { 2 - 4 } & & $\begin{array}{l}\text { (Para todos los instrumentos): } \\
\text { Aquí se explica la relación entre medida de resistencia conversión } \\
\text { a voltaje y conversión a temperatura, presión... } \\
\text { Y otras preguntas similares sobre metrología básica. }\end{array}$ \\
\cline { 2 - 3 } & & $\begin{array}{l}\text { A continuación, se van explicando los diferentes sensores que } \\
\text { componen la estación con ejemplos de lugares con valores } \\
\text { extremos y efemérides. }\end{array}$ \\
\hline
\end{tabular}

TABLA N $^{\circ} 1$. Preguntas que realizan los colaboradores del stand al público para comenzar con la presentación de la Actividad A “Dónde, qué y cómo se mide”.

\begin{tabular}{|c|c|l|}
\hline Actividad & Público & \multicolumn{1}{|c|}{ Preguntas de introducción a la actividad } \\
\hline \multirow{2}{*}{} & & $\begin{array}{l}\text { (Juego de localización de climogramas sobre una imagen } \\
\text { satélite de la superficie terrestre) }\end{array}$ \\
\cline { 2 - 3 } & & $\begin{array}{l}\text { Juego de localización de climogramas con información de } \\
\text { coordenadas geográficas y altitud. La misma actividad se realiza } \\
\text { con diagramas de la Comunidad Autónoma del País Vasco y el } \\
\text { mapa correspondiente.. }\end{array}$ \\
\cline { 2 - 4 } & \multirow{2}{*}{$\begin{array}{l}\text { Esta pregunta se adapta a las diferentes variables climáticas } \\
\text { utilizadas. Se ordenan las columnas de valor para mostrar las } \\
\text { diferentes efemérides y se muestran en papel los modelos de la } \\
\text { situación atmosférica para ese día. } \\
\text { Con las columnas de datos mensuales para cada una de las } \\
\text { variables climáticas se muestran los gráficos seriados con su } \\
\text { línea de tendencia correspondiente. }\end{array}$} \\
\hline
\end{tabular}

TABLA N ${ }^{\circ}$ 2. Preguntas que realizan los colaboradores del stand al público para comenzar con la presentación de la Actividad B “Cómo y para qué se utilizan en Geografía esos datos”. 
Estas actividades de divulgación se realizan utilizando diversos recursos materiales. Atendiendo al primer objetivo planteado, en la Actividad A "Dónde, qué y cómo se mide" se utiliza instrumental facilitado por la Agencia de Meteorología Vasca EUSKALMET: un higrotermotransmisor Rotronic MP103A, un barómetro Setra 270, un pluviómetro Geónica PCP-215, un piranómetro Kipp\&Zonen CM-11, un anemómetro Young 051035 , un sensor de temperatura de suelo Geónica STS, un registrador y controlador Campbell CR1000 y un disdrómetro Parsivel de OTT. Estos sensores en perfecto funcionamiento están acompañados de un ordenador portátil con el software necesario para visualizar, en tiempo real, todas las variables indicadas en la presentación de la actividad. Además, permite tratar cuestiones como el tiempo de respuesta de los sensores, la obtención de medias de cada variable (en general diezminutarias), las necesidades de almacenamiento y transmisión de los datos de todas las estaciones de esa agencia a una sola ubicación y que desde allí se puedan difundir a investigadores y/o público en general. También se dispone de un globo sonda de Vaisala, y diversos sensores fuera de uso y despiezados para mostrar el funcionamiento interno de los mismos (Figura $\mathrm{n}^{\mathrm{o}} 2$ ).

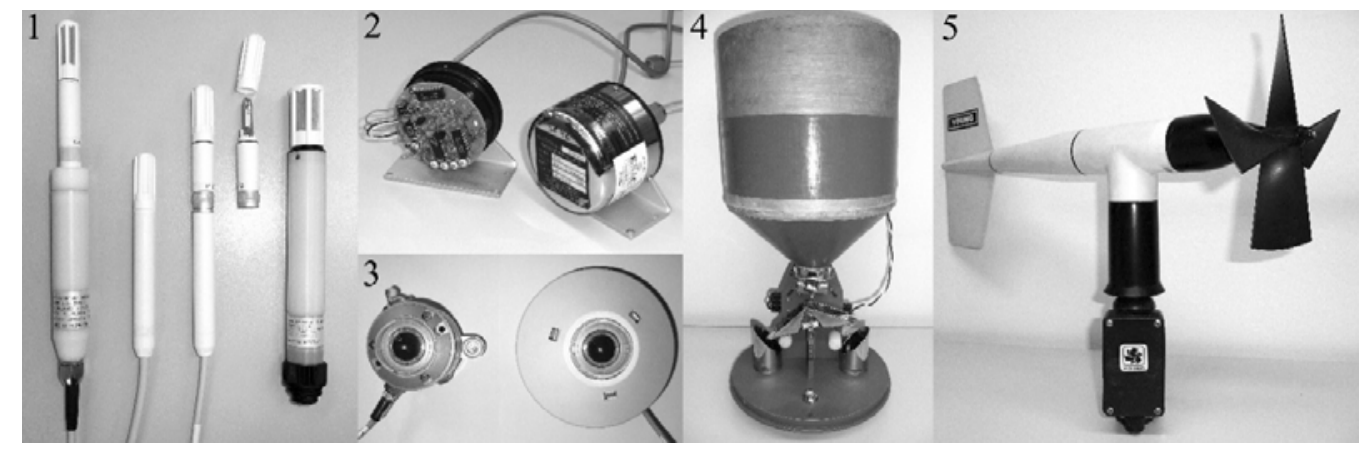

FIGURA N ${ }^{\circ}$ 2. Imagen ilustrativa de algunos de los sensores utilizados en la Actividad A "Dónde, qué y cómo se mide" del stand "Del dato al gráfico climático" (no están a escala) de la SCTI: (1) higrotermotransmisores Rotronic, (2) barómetro Setra, (3) piranómetro Kipp\&Zonen, (4) pluviómetro de cazoletas Geónica y (5) anemómetro Young.

En la consecución del primer objetivo, pero también para alcanzar el segundo y tercer objetivos del proyecto divulgativo, se ha requerido en la Actividad B "Cómo y para qué se utilizan en Geografía esos datos" de los datos mensuales desde que hay registro de los observatorios meteorológicos de los aeropuertos de Vitoria-Gasteiz (código 9087: 19451973 y cód. 9091: 1973-2018) y desde 1981 de los observatorios de Bilbao/Aeropuerto (cód. 1082) y Donostia-San Sebastián/Igeldo (cód. 1024E). Todos ellos suministrados por la Delegación Territorial en el País Vasco de la Agencia Estatal de Meteorología AEMET. También se han utilizado los datos mensuales desde 2001 de las estaciones 
meteorológicas de Altube (42,9\%-2,8 $)$, Añarbe $\left(43,2^{\circ} /-1,8^{\circ}\right)$, Bermeo $\left(43,4^{\circ} /-2,7^{\circ}\right)$, Gorbea $\left(42,9^{\circ} /-2,7^{\circ}\right)$, Herrera $\left(42,5^{\circ} /-2,6^{\circ}\right)$, Iturrieta $\left(42,7^{\circ} /-2,3^{\circ}\right)$, Moreda $\left(42,5^{\circ} /-2,4\right.$ y con datos desde 2013$)$, Salvatierra $\left(42,8^{\circ} /-2,3^{\circ}\right)$ y Zambrana $\left(42,6^{\circ} /-2,8^{\circ}\right)$ pertenecientes a la Agencia Vasca de Meteorología EUSKALMET. Esta información ha sido recopilada (http://opendata.euskadi.eus/) y tratada en un documento Excel para la realización de la Actividad B y la elaboración de diferentes gráficos climáticos (a igual escala) que se han impreso y plastificado para la ejecución del juego de localización de climogramas -segundo objetivo- (Figura $\left.n^{\circ} 3\right)$.

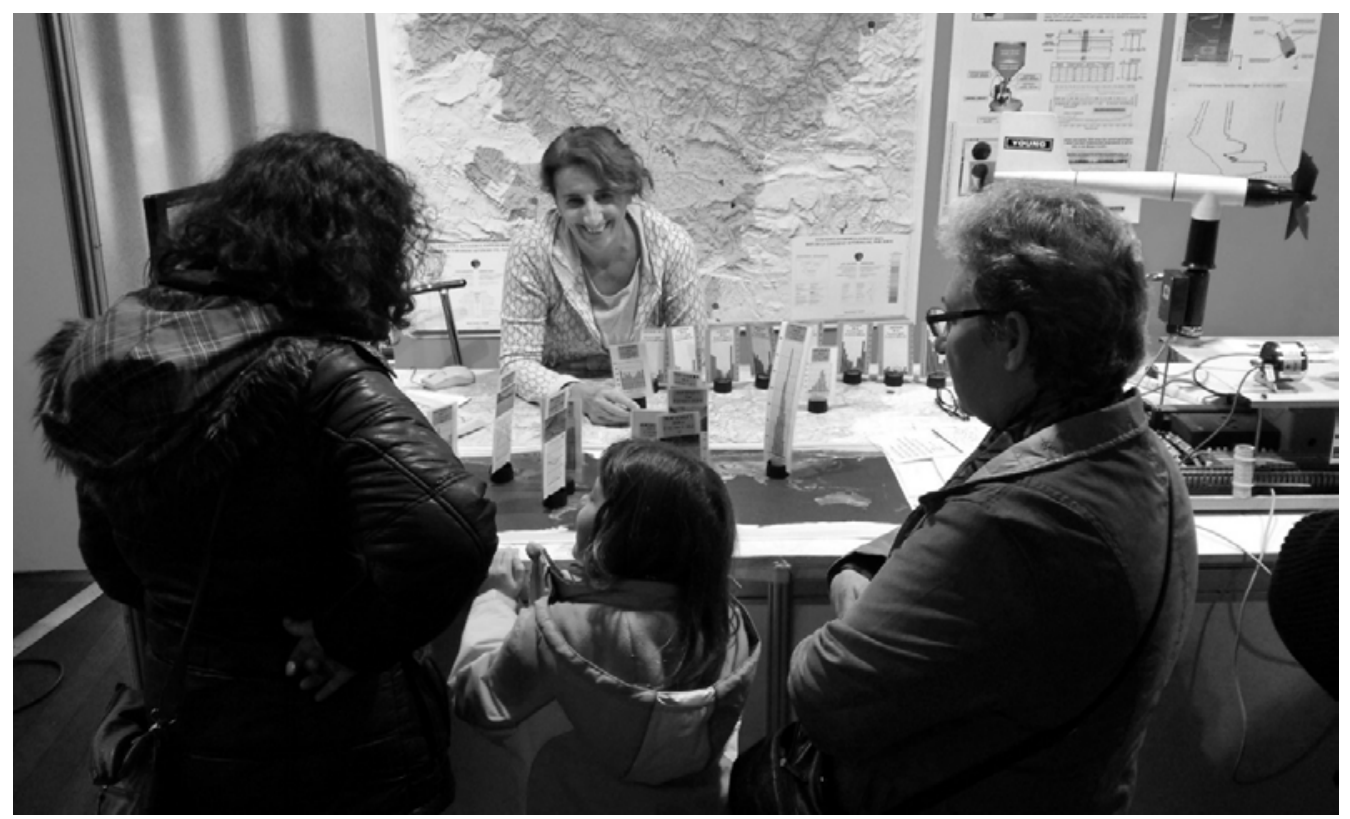

FIGURA $\mathrm{N}^{\mathrm{O}}$ 3. Imagen ilustrativa de los climogramas y los mapas e imagen satélite utilizados para la escala mundial en la Actividad B del stand "Del dato al gráfico climático" de la SCTI.

Además, para las diferentes escalas del juego, mundial y autonómica, se ha utilizado un mapa mural en relieve de la CAPV y facilitado por la Dirección de Planificación Territorial Urbanismo y Regeneración Urbana del Gobierno Vasco y una imagen satélite de la superficie terrestre. En esa Actividad B también se ha utilizado un ordenador con el fin de:

- mostrar los datos diezminutarios y diarios de las diversas estaciones meteorológicas y disponibles en la página de la Agencia Vasca de Meteorología EUSKALMET (http://www.euskalmet.euskadi.eus/) y las figuras de mapas, modelos y gráficos correspondientes (Figura $\left.n^{\circ} 4\right)$; 


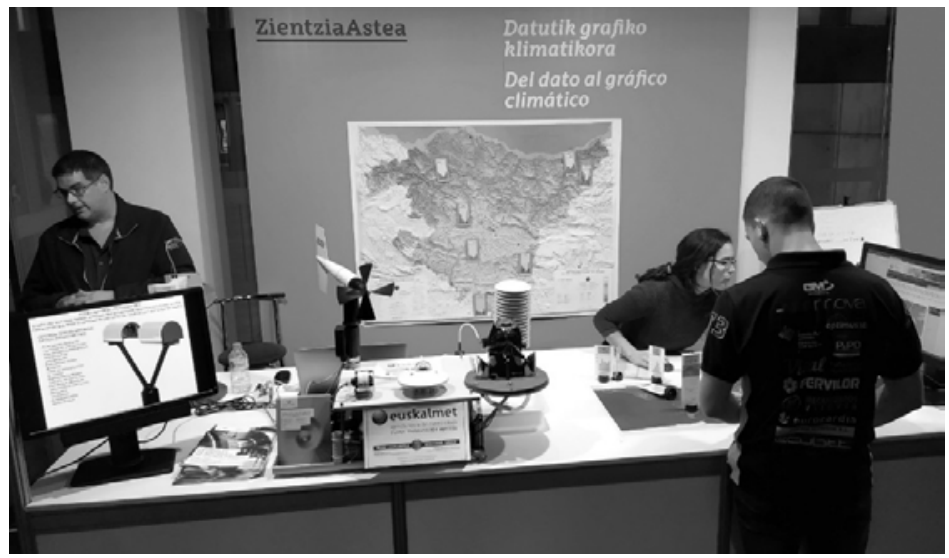

FIGURA $N^{\circ}$ 4. Imagen que recoge parte del material utilizado en el stand "Del dato al gráfico climático" de la SCTI.

- presentar un documento PowerPoint que recoge de manera sucinta y gráfica un resumen de los factores que determinan la diversidad climática mundial (Figura $\mathrm{n}^{\circ}$ 5) y que ayuda a interpretar y responder adecuadamente en el juego de localización;
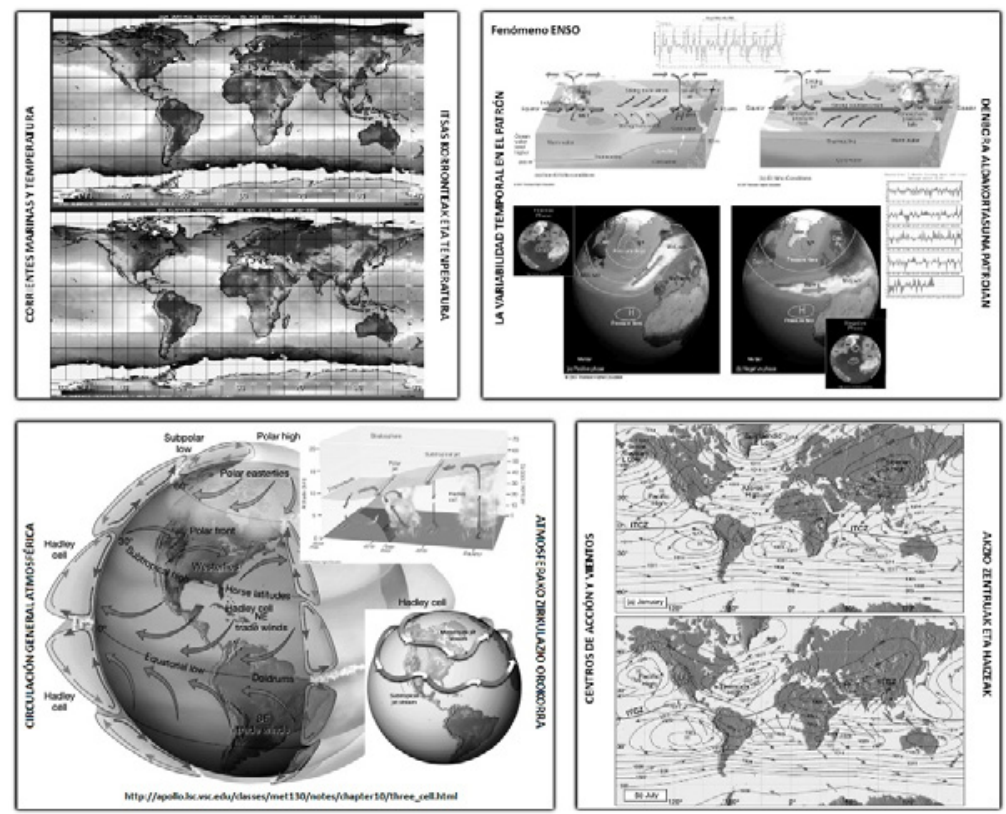

FIGURA $\mathrm{N}^{\mathrm{o}}$ 5. Ejemplos de la información gráfica utilizada en el documento PowerPoint para explicar y mostrar varios de los factores que influyen en la diversidad climática. 
- presentar un documento Excel que contiene datos mensuales de la serie climática de Vitoria-Gasteiz (temperatura media mensual, media mensual de la temperatura máxima diaria, media mensual de la temperatura mínima diaria, temperatura máxima absoluta mensual y día, temperatura mínima absoluta mensual y día, número de días de helada de cada mes, precipitación mensual, precipitación máxima diaria mensual y día, número de días de lluvia, de nieve y de granizo de cada mes);

- mostrar información gráfica sobre cómputos extremos (Moncho, Bella y Caselles, 2011, p. 122) (Figura ${ }^{\circ} 6$ ) y sobre los gráficos de modelos que permiten explicar los factores que determinan un tipo de tiempo con registros significativos en alguna de las variables atmosféricas $\left(\right.$ Figura $^{\circ} 7$ );

\begin{tabular}{|c|c|c|c|}
\hline TIEMPO/DENBORA & $\begin{array}{l}\text { PRECIPITACIÓN/ } \\
\text { PREZIPITAZIOA }\end{array}$ & ESTACIÓN/BEHATOKIA & FECHA/DATA \\
\hline $1 \mathrm{~min}$ & 38,1 & Barot (Guadalupe) & $26 / 11 / 1970$ \\
\hline $8 \mathrm{~min}$ & 126 & Füssen (Alemania) & $25 / 05 / 1920$ \\
\hline $15 \mathrm{~min}$ & 198 & Plumb Poin (Jamaica) & $12 / 05 / 1916$ \\
\hline $20 \mathrm{~min}$ & 206 & Curtea-de-Arges (Rumania) & $07 / 07 / 1889$ \\
\hline $30 \mathrm{~min}$ & 280 & Sikeshugou (China) & $03 / 07 / 1974$ \\
\hline $42 \mathrm{~min}$ & 305 & Holt, Misuri (EEUU & $22 / 06 / 1947$ \\
\hline $60 \mathrm{~min}$ & 401 & Shangai (Mongolia) & $03 / 07 / 1975$ \\
\hline $72 \mathrm{~min}$ & 440 & Gaoj, Gansu (China) & $12 / 08 / 1985$ \\
\hline 2 horas & 489 & Yujiawanzi (Mongolia) & $19 / 07 / 1975$ \\
\hline $2,5 \mathrm{~h}$ & 550 & Bainaobao (China) & $25 / 06 / 1972$ \\
\hline $3 \mathrm{~h}$ & 600 & Duan Jiazhuang (China) & 28/07/1977 \\
\hline $6 \mathrm{~h}$ & 840 & Muduocaidang (Mongolia) & $01 / 08 / 1977$ \\
\hline $9 \mathrm{~h}$ & 1087 & Belouve (La reunión) & $28-29 / 02 / 1964$ \\
\hline $10 \mathrm{~h}$ & 1400 & Muduocaidang (Mongolia) & 01/08/1977 \\
\hline $12 \mathrm{~h}$ & 1340 & Belouve (La Reunión) & $28-29 / 02 / 1964$ \\
\hline $18,5 \mathrm{~h}$ & 1689 & Belouve (La Reunión) & $28-29 / 02 / 1964$ \\
\hline $20 \mathrm{~h}$ & 1697 & Foc-Foc (La Reunión) & $7-8 / 01 / 1966$ \\
\hline $22 \mathrm{~h}$ & 1780 & Foc-Foc (La Reunión) & $7-8 / 01 / 1966$ \\
\hline $24 \mathrm{~h}$ & 1870 & Cilaos (La Reunión) & $15-16 / 03 / 1952$ \\
\hline 2 días & 2467 & Aurère (La Reunión) & $7-8 / 04 / 1958$ \\
\hline $3 d$ & 3929 & Commerson (La Reunión) & $24-26 / 02 / 2007$ \\
\hline $4 d$ & 4869 & Commerson (La Reunión) & $24-27 / 02 / 2007$ \\
\hline $8 \mathrm{~d}$ & 4936 & Commerson (La Reunión) & $20-27 / 01 / 1980$ \\
\hline $9 \mathrm{~d}$ & 5342 & Commerson (La Reunión) & $19-27 / 01 / 1980$ \\
\hline $10 d$ & 5678 & Commerson (La Reunión) & $18-27 / 01 / 1980$ \\
\hline $11 \mathrm{~d}$ & 5949 & Commerson (La Reunión) & $17-27 / 01 / 1980$ \\
\hline $12 \mathrm{~d}$ & 6051 & Commerson (La Reunión) & $16-27 / 01 / 1980$ \\
\hline 1 mes & 9300 & Cherrapunji (India) & $07 / 1861$ \\
\hline 2 mes & 12767 & Cherrapunji (India) & $06-07 / 1861$ \\
\hline 1 año & 26461 & Cherrapunji (India) & $08 / 1860-09 / 1861$ \\
\hline
\end{tabular}

FIGURA $N^{0} 6$. Datos de extremos de precipitación mundiales utilizados para realizar la comparativa con los datos registrados en la Comunidad Autónoma Vasca. Fuente: Moncho, Bella y Caselles, 2011, p. 122. 

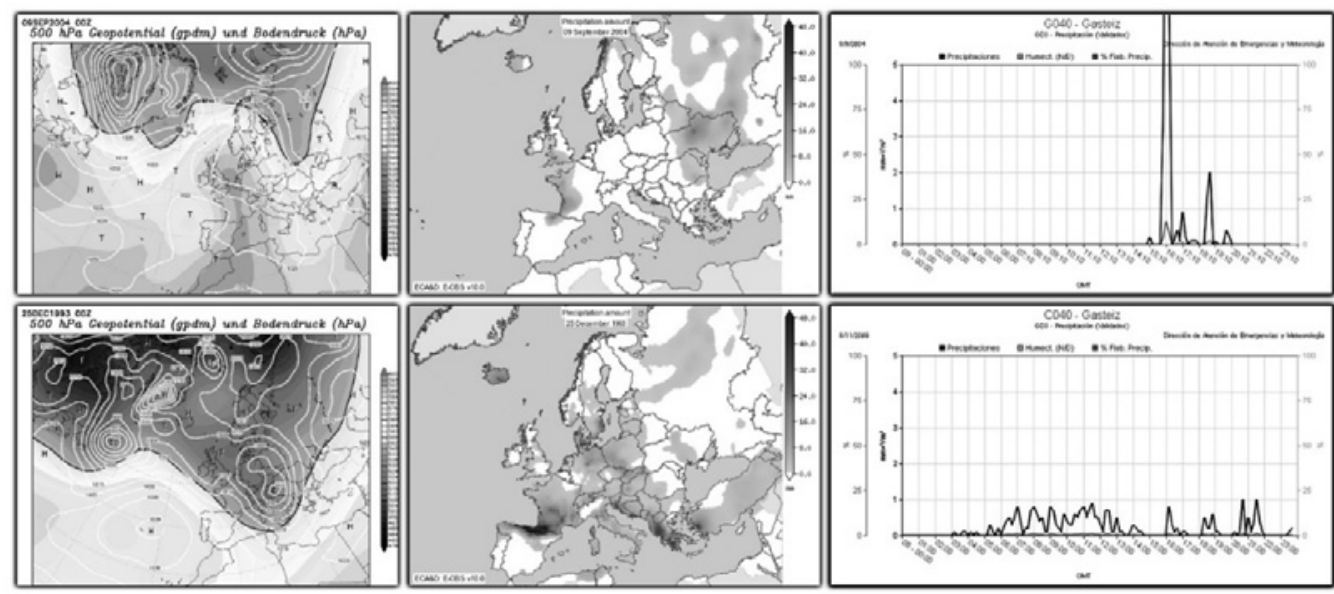

FIGURA $\mathrm{N}^{\mathrm{0}}$ 7. Ejemplo de imágenes de modelos y gráficos utilizados para ilustrar y presentar los factores que determinan en los registros de los observatorios cómputos elevados para las diferentes variables atmosféricas. Fuentes: http://www.wetterzentrale.de/, http://www.

euskalmet.euskadi.eus

- posibilitar al público el acceso a diferentes páginas de internet sobre datos meteorológicos, marinos y predicción:

- http://worldweather.wmo.int/en/home.html

- https://aquarius.nasa.gov/

- https://www.ncdc.noaa.gov/cag/global/time-series

- https://earth.nullschool.net/

- http://www.argo.ucsd.edu/

- http://www.wetterzentrale.de/.

- Por último, en cada una de las ediciones se ha ido preparando un poster sobre diferentes variables y elementos climáticos. En ellos se contextualizan el rango de variabilidad de los mismos y los distintos métodos de medida utilizados en la ciencia meteorológica, tanto manuales como automáticos. Además, para amenizar el tiempo de espera y atraer a los visitantes de la SCIT, a partir de ellos se han editado videos que son proyectados en los monitores del stand. Los posters elaborados y expuestos tratan sobre:

- (Figura $\left.n^{\circ} 8\right)$. En él se puede observar la variación de presión desde el centro de la Tierra hasta la Exosfera y se comparan valores de presión: el necesario para producir un diamante, el que tendríamos en la cima del Everest, en el interior de un neumático de coche... Además, con el fin de explicar las diferentes unidades 
que se pueden utilizar para expresar medidas de la misma magnitud, se confrontan las unidades del sistema internacional (SI) para presión (Pascal, Pa) con la unidad utilizada en meteorología (milibar, mbar);
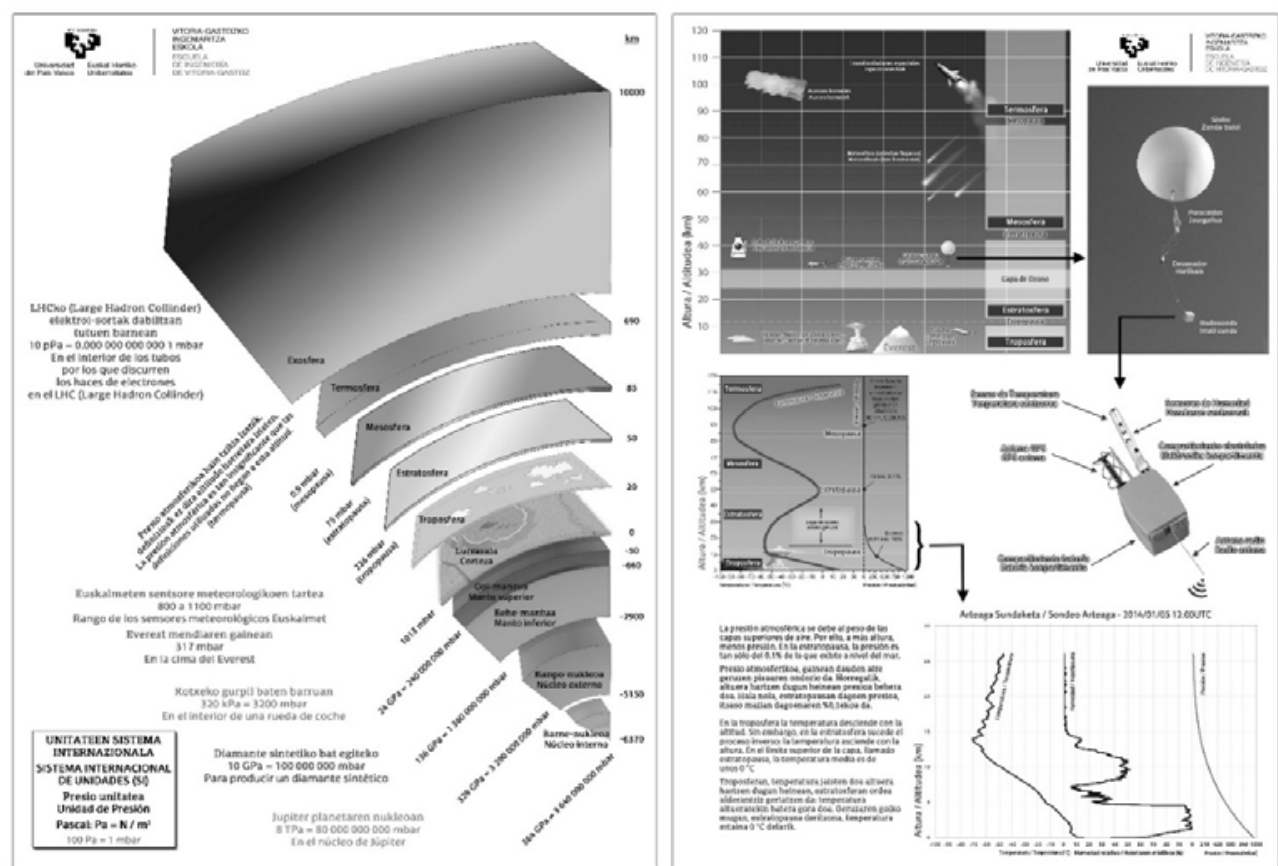

FIGURA N ${ }^{\circ} 8$. Ejemplos de posters sobre la presión y sobre los globos sonda elaborados para el stand "Del dato al gráfico climático" de la SCTI.

- (Figura $\mathrm{n}^{\mathrm{o}} 8$ ). En él se muestra la obtención de diversos parámetros utilizando globos sonda y la variabilidad de los mismos con la altura. Se confrontan datos obtenidos de la estación de Gautegiz-Arteaga (EUSKALMET) con las medidas más teóricas e incidiendo en la dificultad de estos globos para alcanzar alturas superiores a $30 \mathrm{~km}$.

- (Figura $n^{\circ} 9$ ). En él se representan mediante tres gráficos circulares a la misma escala, el volumen total de agua existente en nuestro planeta, el agua dulce y el tamaño de la tierra. Otro esquema segmenta el total de agua en los distintos estados existentes, de forma que se resalta la relativamente pequeña cantidad de agua dulce y la importancia del agua en forma de vapor en la atmósfera. Este poster también permite explicar el origen de la unidad de medida "', el carácter discontinuo de la precipitación de lluvia, las dificultades para su medida o la representatividad de la misma; 


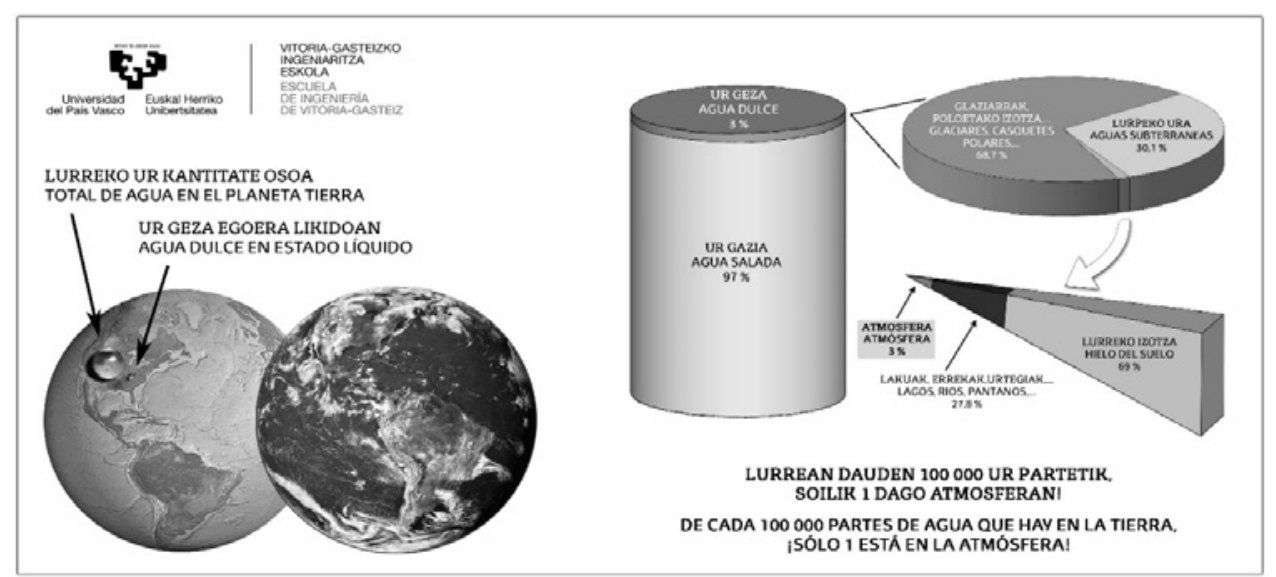

FIGURA $\mathrm{N}^{\mathrm{o}}$ 9. Detalle poster referido al agua en la tierra y en el que queda reflejado el pequeño volumen del agua dulce respecto del agua salada. Fuente: Howard Perlman, USGS; Globe Illustration by Jack Cook, Woods Hole Oceanographic Institution (C); Adam Nieman.

Por su parte, los recursos humanos con los que ha contado el stand para la realización de las actividades han sido profesores del área de conocimiento de Geografía Física del Departamento de Geografía y del Departamento de Tecnología Electrónica, así como técnicos del Laboratorio de Calibración de la Escuela de Ingeniería de VitoriaGasteiz. Además, cada año han colaborado en el stand alumnos de $4^{\circ}$ curso del Grado de Geografía y Ordenación del Territorio y posgrados.

\section{RESULTADOS}

La experiencia de organización de un stand en la Semana de la Ciencia, la Tecnología y la Innovación en la UPV/EHU atendiendo a los objetivos planteados ha tenido como resultado el acercamiento a la ciudadanía de información científica de carácter tecnológico como causal y descriptivo; mostrando en un único stand cómo la investigación en una ciencia, en nuestro caso la geográfica, requiere de bases de datos recogidas y aportadas tanto, por otras ramas tecnológicas y de conocimiento como, por entidades diversas. Estos hechos pueden ser expresados a partir de una actividad de interactuación con quienes visitan el stand.

\subsection{Divulgar sobre tecnología y ciencia geográfica a través de un proyecto colaborativo}

Respecto a la Actividad A "Dónde, qué y cómo se mide", la presencia física de una estación meteorológica y su presentación y explicación han permitido comprender el 
funcionamiento y la magnitud de la infraestructura necesaria para obtener datos fiables. La disponibilidad de sensores fuera de uso ha servido para visualizar de cerca la naturaleza de los transductores, facilitando de esta manera la comprensión de la explicación técnica. Se han aprovechado investigaciones y programas internacionales que están disponibles en internet para divulgar otros parámetros que se obtienen a nivel planetario y que ayudan a modelizar mejor el estado de la atmósfera. Durante las visitas y en función de la edad, el conocimiento previo, el interés, etc. se han tratado los siguientes aspectos relacionados con la metrología meteorológica:

- importancia de la calibración en la obtención de series temporales coherentes;

- acreditación de los laboratorios que calibran los sensores meteorológicos;

- relativización del dato: la importancia de conocer la incertidumbre de cada medida y la influencia de ésta en los modelos, o a la hora de utilizar dichos datos;

- valor de los metadatos, esos compañeros desconocidos de la medida que aportan calidad a la misma y que habitualmente se obvian;

- ventajas de las estaciones meteorológicas automáticas en la gestión de riesgos por precipitaciones intensas.

En la Actividad B “Cómo y para qué se utilizan en Geografía esos datos” (y siempre en función del público presente en el stand) se han abordado diferentes temáticas de la disciplina geográfica relacionadas con la climatología:

- el dato del sensor verificado y el cómputo total y medio diario, mensual y anual de las variables que permiten mostrar la distinción entre los conceptos de Tiempo atmosférico y Clima y entre Meteorología y Climatología;

- el análisis de los principales factores y procesos que intervienen en la variabilidad climática espacial, estacional y anual: variables y dinámica atmosférica en las diferentes latitudes condicionada por mares, relieves y continentes y los fenómenos de la Oscilación del Sur-El Niño (ENSO) y la Oscilación del Atlántico Norte (NAO);

- la interpretación de la diversidad climática mundial atendiendo a dos descriptores: los cómputos medios anuales de temperatura y precipitación y sus regímenes mensuales en comparación con los datos de Vitoria-Gasteiz: algunos de los lugares más lluviosos (Puerto López, Colombia 2,8 /-77,23; Cherrapundji, India- 25,3 191,7º; Milford Sound, Nueva Zelanda -44,6\%167,9\%; Monte Waialeale, Islas Hawai 22,06\%-159,5\%; Douala, Camerún 40,3\% $/ 9,46^{\circ}$ ) y más secos (Vostok, Rusia $-78,45^{\circ} / 106,8^{\circ}$; Iquique, Chile $-20,2^{\circ} /-70,1^{\circ}$; Death Valley, USA- 36,5\%-116,9\%; 
Aswan, Egipto $24,09^{\circ} / 32,8^{\circ}$; Walvis Bay, Namibia $-22,9^{\circ} / 14,5^{\circ}$ ) del mundo, los factores determinantes y las características de la distribución estacional de valores de temperatura y precipitación, así como de los valores térmicos y pluviométricos medios anuales;

- el análisis de la variabilidad climática en la Comunidad Autónoma del País Vasco y los principales factores que intervienen en la misma. Los datos de casi 20 años de registro de las estaciones de EUSKALMET y de más de 30 años de AEMET han permitido elaborar un amplio número de climogramas que muestran la diversidad de cómputos y reparto de la precipitación, valores medios anuales de temperatura y amplitud térmica, meses secos, diferencias en el comportamiento de los valores medios mensuales de las temperaturas mínimas y máximas diarias en función de la cercanía al mar, la altitud o la continentalización;

- la importancia de la utilización de series largas de datos para la caracterización óptima de los patrones climáticos: esta actividad se ha realizado utilizando el climograma de la estación meteorológica de Moreda que con 6 años únicamente de registro no representa adecuadamente el patrón que corresponde a este ámbito (Figura $\mathrm{n}^{\mathrm{o}} 10$ ).
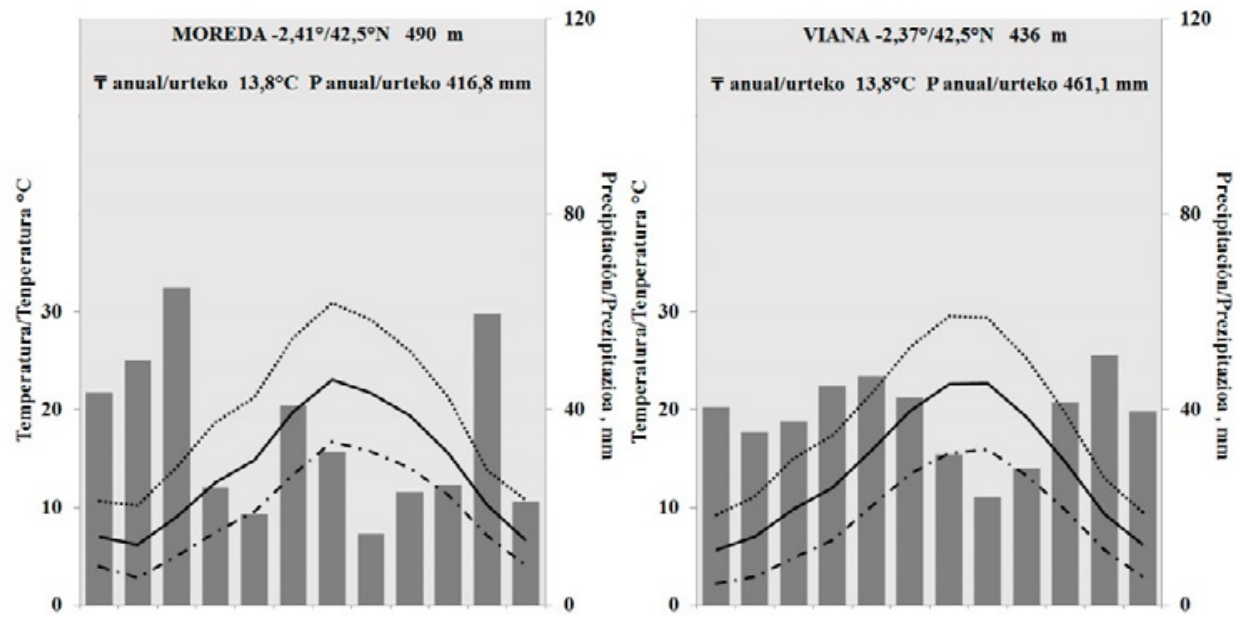

FiguRA $N^{\circ} 10$. El climograma de la estación de Moreda (izquierda) que solo cuenta con 6 años de registro muestra un mayor número de meses de carácter seco (mayo, julio, agosto, septiembre y octubre) que el que corresponde a este ámbito climático (julio, agosto y septiembre), con un montante de precipitación de enero a marzo superior y un valor medio mensual de las temperaturas mínimas diarias en invierno también superior a los promedios constatados para series más largas (Ruíz y Galdos, 2008) en estaciones meteorológicas cercanas (derecha, ejemplo de la estación de Viana con datos desde 1982). 
- el rigor en la utilización y en la transferencia de la información de interés general: la relación entre percepción personal y mediatizada y los datos de registro. Confirmación, con datos verificados y desde que hay registro para la ciudad de Vitoria-Gasteiz, de que si, como habitualmente se percibe y dice, " (comparativa cronológica con el cómputo de días de nieve mensual y anual), “ (comparativa cronológica con la cantidad de precipitación registrada mensualmente y su línea de tendencia), “(comparativa cronológica en temperatura media mensual, media mensual de la temperatura máxima diaria, media mensual de la temperatura mínima diaria, temperatura máxima absoluta mensual, temperatura mínima absoluta mensual y sus líneas de tendencia) o " (número de días de helada mensual).

\subsection{Interactuar de forma amena con la ciudadanía con el fin de interesar en la práctica tecnológica y geográfica}

La preparación de los materiales para componer la información que podía ser utilizada en el stand ha consistido en un trabajo arduo condicionado por la diversidad de quienes pueden acercarse al stand a interesarse por el contenido del mismo. Por ello, en función del tipo de público, este material se ha mostrado de forma adaptada y modificando el relato y las características de la actividad.

En la Actividad A "Dónde, qué y cómo se mide”, observar la "carcasa y las entrañas" de cada sensor, "regar" agua en el pluviómetro para percibir cómo vuelcan las cazoletas, presionar con los dedos un "filamento" para comprobar gráficamente en el ordenador como sube la temperatura, soplar frente al anemómetro para determinar la velocidad del "viento", hacernos una idea del volumen que ocupa la lluvia que cae en un año en VitoriaGasteiz y compararla con la que cae en otros lugares del mundo o adivinar el nombre de aquello que parece un "platillo volante" y es un radiómetro, ha sido una experiencia de acercamiento e información sobre instrumentos y tecnología para el público en general que ha mostrado su gratitud por ver y entender una estación meteorológica. Pero también ha sido una experiencia de conocimiento para aquellas personas más especializadas a las que se les ha explicado la diferencia entre "calibrar" y "ajustar" o que ${ }^{\circ} \mathrm{C}$ significa grado Celsius y no grado centígrado; o cómo se efectúa la calibración de los distintos sensores; o cuál es el principio físico que se utiliza para determinar la temperatura en los sensores automáticos utilizados por EUSKALMET, que no es otro que, la medida de la variación de una resistencia de platino que a su vez se amplifica y se convierte en una variación de tensión, de forma que una variación entre $-40{ }^{\circ} \mathrm{C}$ y $60{ }^{\circ} \mathrm{C}$ se convierte en una variación de tensión continua entre $0 \mathrm{~V}$ y $1 \mathrm{~V}$. También han sido tratados temas como la problemática de la medición automática que se ve compensada con la inmensidad de los datos recopilados y la estadística, etc. 
En la Actividad B “Cómo y para qué se utilizan en Geografía esos datos”, comparar los datos diezminutarios del momento con los modelos de superficie y altura y radiosondeos; observar los datos medios y absolutos mensuales de series largas para, en unos casos determinar efemérides y analizar sus causas mediante la consulta de los modelos correspondientes a ese día y en otros casos, conocer el patrón de cada uno de los parámetros atmosféricos y observar su variabilidad temporal; informarse sobre aspectos como los efectos en la dinámica atmosférica de las corrientes cálidas y frías de los mares o de la intensidad y dirección de los gradientes de presión y sus oscilaciones; observar en mapas sintéticos la distribución espacial y temporal de diversas variables atmosféricas; interpretar climogramas y saber localizarlos en la escala mundial y autonómica o simplemente hacer corresponder las manchas de colores y formas de la imagen de la pantalla del ordenador con la imagen en la mesa expositora en el caso de los niños más pequeños, ha resultado una práctica de acercamiento al quehacer geográfico realizada de forma interactiva y amena. El recurso a la experiencia personal en el recuerdo de efemérides, de comparativas numéricas contrastadas gráficamente y de validar información o conocimiento particular a través de los juegos de identificación y localización, ha sido una labor didáctica realizada por los colaboradores en el stand que ha tenido como recompensa la sonrisa y el agradecimiento expreso de quienes han participado en la actividad.

Por último y para el tercero de los objetivos, mostrar a los ciudadanos la necesidad y el valor de la colaboración en el trabajo científico, el ver unidos en un mismo stand dos centros diferentes de enseñanza e investigación universitaria y a personas de diferentes ramas de conocimiento colaborando ha permitido visibilizar el hecho de la complementariedad como fortaleza del quehacer educativo y científico (Figura $\mathrm{n}^{\mathrm{o}} 11$ ).

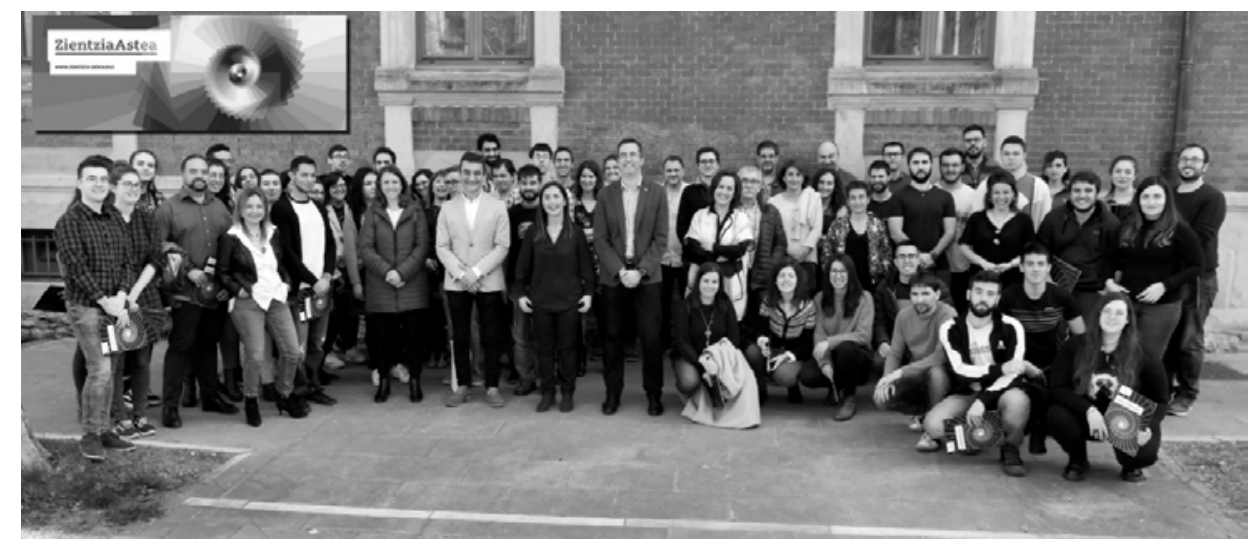

FigURA N $^{0} 11$. Grupo de colaboradores de la XVII Semana de la Ciencia, la Tecnología y la Innovación (2018) del Campus de Álava en el acto de reconocimiento de la actividad por parte de los representantes de la UPV/EHU. 


\section{CONCLUSIONES Y DISCUSIÓN}

A través de las actividades divulgativas como la que aquí se ha presentado, el stand “Del dato al gráfico climático", la Semana de la Ciencia, la Tecnología y la Innovación, SCTI es una oportunidad de aprendizaje no formal para los alumnos en los diferentes niveles educativos, que entronca en la actividad aquí presentada con contenidos imprescindibles dentro de los currícula de Geografía (Tonda y Sebastiá, 2003) y similar a otras propuestas museísticas (Morote y Moltó, 2017) o de talleres en el aula (Cruz, 2010). En este sentido, cabe señalar que habitualmente el profesorado de los centros educativos inscritos que acompaña a los grupos escolares en la SCTI es el de ciencias y por tanto son los alumnos de esta rama de conocimiento quienes visitan los stands. Por ello se insiste en la necesidad de que también los docentes de otras áreas de conocimientos se hagan eco de este evento y acudan al mismo con el alumnado de sus asignaturas. En nuestro caso, las actividades del stand pueden ser un recurso educativo innovador para los docentes de las asignaturas de Ciencias Sociales de Educación Primaria, Geografía e Historia de la ESO y de Geografía de España de $2^{\circ}$ de Bachillerato (Martínez y Olcina, 2019). Pero también es una ocasión para transferir, a la escala que le compete, conocimiento al conjunto de la sociedad. De ahí la importancia, como se ha referido en el apartado de resultados, de la capacidad de los responsables del stand para adecuar el discurso y los materiales desarrollados para su presentación a un público diverso.

En el tiempo que ha durado la experiencia divulgativa se han modificado y adaptado las presentaciones como los recursos gráficos de las dos actividades en respuesta a la motivación mostrada por los participantes en el stand y las sugerencias recibidas por su parte. Además, se han ido incorporando nuevas fuentes de consulta meteorológica y climática como visual. Pero ciertamente, la experiencia realizada no ha contado con un diagnóstico sobre satisfacción del público a partir de la elaboración de un cuestionario y por tanto lo recogido en este artículo únicamente expresa la valoración cualitativa de los autores. Las características del evento organizado y coordinado por el Vicerrectorado de Desarrollo Científico y Transferencia, masivo y abierto a todo tipo de público (3.200 personas en 2018, http://zientzia-astea.org/es/zientzia-astea-vitoria-gasteiz/), con numerosos stands, talleres y otras actividades, consideramos no propician la realización de cuestionarios de satisfacción. En cambio, sí resultaría pertinente para posteriores ediciones la realización de una encuesta dirigida a los alumnos de Educación Obligatoria Secundaria y Bachillerato (22 centros escolares en 2018) una vez finalizada su visita a la SCIT y que permitiera, además de conocer la idoneidad de las actividades propuestas su relación con las competencias adquiridas en el currículo educativo.

Por otra parte, se trata de una oportunidad para la difusión y proyección de la Geografía. Una ciencia que, aunque se ocupa de temáticas de interés actual y general, como disciplina escolar ha sufrido en los últimos años una notable reducción de su presencia 
en el currículo (Olcina, Zúñiga y De Miguel, 2018). La Comunidad Autónoma del País Vasco no es ajena a esta situación. A pesar de que, como recoge el texto Refundido del Decreto por el que se establece e implanta el currículo de Bachillerato (Decreto127/2016, p. 118), la Geografía "es una ciencia que contribuye a la comprensión e interpretación de lo que sucede en el planeta y en nuestro entorno inmediato; socialmente útil ya que estudia y analiza problemáticas actuales...”, ya han sido constatados los perjuicios de su escasa consideración en las Pruebas de Acceso a la Enseñanza Universitaria (Ormaetxea, 2018). En esta situación, como geógrafos docentes e investigadores, en esta labor de transferencia a través del stand, estamos realizando también un trabajo de visibilización que consideramos, es fundamental.

Por último, queremos insistir en el valor de la colaboración entre ramas de conocimiento. La Geografía necesita para el análisis territorial, del dato cuantitativo aportado, entre otras, por las tecnologías paramétricas y de fuentes diversas. Mostrar a la sociedad esta acción colaborativa a través del stand supone desde nuestra perspectiva un hito pedagógico.

\section{AGRADECIMIENTOS}

La realización de la actividad divulgativa ha contado con la aportación del Vicerrectorado de Investigación (2014-15) y del Vicerrectorado de Desarrollo Científico y Transferencia (2016-18) de la UPV/EHU. Nuestro agradecimiento expreso a los colaboradores en el stand: Jon Cadierno, Adrián Díez, Paula Pérez, José Miguel-García y June Ollacarizqueta.

\section{BIBLIOGRAFÍA}

Cruz, L.A., 2010. "Diseño de un taller de meteorología para alumnos de educación secundaria” en M.J. Marrón, coord. Geografía, educación y formación del profesorado en el marco del Espacio Europeo de Educación Superior. Madrid: Asociación de Geógrafos Españoles-Grupo de Didáctica de la Geografía (AGE), pp. 215-236. [En línea] Disponible en: <https://www.age-geografia.es/ didacticageografia/docs/Publicaciones/2010_Formacion_profesorado_EEES1. pdf>. [Último acceso 3 septiembre 2019]

Decreto 127/2016, de 6 de septiembre, por el que se establece el currículo de Bachillerato y se implementa en la Comunidad Autónoma del País Vasco. Boletín Oficial del País Vasco. Vitoria-Gasteiz, 23 de septiembre de 2016, nº 182, pp. 1-300. [En línea] Disponible en: <http://www.euskadi.eus/desarrollo-curricular/web01-a3hakade/ es/>. [Último acceso 3 marzo 2019] 
Del Campo, M ${ }^{a}$ D., 2018. ¿Sabías que ${ }^{\circ} \mathrm{C}$ significa grado Celsius y no grado centígrado? e-medida. Revista Española de Metrología. [En línea. Acceso Libre]. Madrid: Centro Español de Metrología, Volumen 7, no 14, diciembre. <https://www.emedida.es/numero-2/oc-significa-grado-celsius-y-no-grado-centigrado/>. [Último acceso 28 febrero 2019]

Fernández, I.; Mecha, R. y Milán, M., 2018. La Comunidad Científica ante los Medios de Comunicación. Guía de Actuación para la Divulgación de la Ciencia, Universidad Complutense de Madrid, 36 pp. [En línea] Disponible en: <https://www.ucm.es/ data/cont/docs/1334-2018-05-04-Guia de actuación WEB.pdf>. [Último acceso 28 enero 2019]

Ruíz, E. y Galdos, R., 2008. Geografía del País Vasco. Donostia-San Sebastián: Editorial Nerea.

Martínez,L.C.; Olcina,J., 2019. "La enseñanza escolar del tiempo atmosférico y del clima en España: currículo educativo y propuestas didácticas". Anales de Geografía de la Universidad Complutense, 39(1), pp. 125-148. [En línea] Disponible en: <https:// revistas.ucm.es/index.php/AGUC/article/view/64680/45644565513459>. [Último acceso 3 septiembre 2019]

Mecha, R., 2010. "Formación geográfica en los grandes eventos de cultura científica". Didáctica Geográfica, 11, pp. 17-35. [En línea] Disponible en: <http://www. age-geografia.es/didacticageografica/index.php/didacticageografica/article/ viewFile/39/39>. [Último acceso 3 septiembre 2019]

Moncho, R.; Belda, F. y Caselles, V., 2011. "Distribución probabilística de los extremos globales de precipitación". Nimbus: Revista de Climatología, meteorología y paisaje, 27-28, pp. 119-135. [En línea] Disponible en: <http://repositorio.ual.es/ handle/10835/1537>. [Último acceso 3 octubre 2018]

Morote, A. F. y Moltó, E., 2017. "El Museo del Clima de Beniarrés (Alicante). Propuesta de un recurso didáctico para la enseñanza de la Climatología”. Didáctica de las ciencias experimentales y sociales, 32, 1, pp. 109-131. [En línea] Disponible en: < https://ojs.uv.es/index.php/dces/article/view/9624>. [Último acceso 2 septiembre 2019]

Olcina, J.; Zúñiga, M. y De Miguel, R., 2018. Aportación conjunta de las tres entidades representativas de la Geografía española ante consulta pública previa sobre un Anteproyecto de Ley Orgánica de Modificación de la Actual Ley de Educación, Asociación de Geógrafos Españoles, 2 pp. [En línea. Acceso Libre] Disponible en: <https://www.age-geografia.es/site/wp-content/uploads/2018/11/ APORTACION-CONJUNTA-MODIFICACION-DE-LA-ACTUAL-LEY-DEEDUCACI\%C3\%93N.pdf>. [Último acceso 11 febrero 2019] 
Ormaetxea, O., 2018. Informe sobre los parámetros de ponderación de Geografía en la universidad del país vasco y su relación con las universidades públicas españolas (curso 2017/18),Asociación de GeógrafosEspañoles,30pp.[Enlínea.AccesoLibre] Disponible en: <https://www.age-geografia.es/site/wp-content/uploads/2018/06/ INFORME-PAR \% C 3\%81 METROS-DE-PONDERACI\%C 3\%93NUNIVERSIDADES-P\%C3\%9ABLICAS-ESPA\%C3\%91OLAS-1.pdf> [Último acceso 11 febrero 2019]

Orruño, M.; Bikandi, J.; San Millán, R. y Arana, I., 2015. "Zientzia Astea de la UPV/ EHU: una oportunidad de difundir la microbiología”. Sem@foro, 59, pp. 3637. [En línea] Disponible en: < https://www.semicrobiologia.org/secciones/ publicaciones/semaforo $>$ [Último acceso 1 febrero 2019]

Tonda, E.Ma .; Sebastiá, R., 2003. "Las dificultades en el aprendizaje de los conceptos de tiempo atmosférico y clima: la elaboración e interpretación de climogramas". Revista de Educación de la Universidad de Granada, 16, pp. 47-69. [En línea] Disponible en: < https://revistas.ucm.es/index.php/AGUC/article/view/64680>. [Último acceso 3 septiembre 2019]

Ramos, J.M.; Calonge, G., 2014. "Renovación de la didáctica de la Geografía Física en $2^{\circ}$ de Bachillerato. La climatología como ejemplo". Didáctica Geográfica, 14, pp. 129-151. [En línea] Disponible en: <http://www.age-geografia.es/ didacticageografica/index.php/didacticageografica/article/view/270>. [Último acceso 3 septiembre 2019]

Universidad del País Vasco/Euskal Herriko Unibertsitatea, 2011. UPV/EHUko Estatutoak. Estatutos de la UPV/EHU, Servicio Editorial de la Universidad del País Vasco, pp.10-11. [En línea] Disponible en: <https://www.ehu.eus/ documents/3026289/3106907/Estatutos_UPV_EHU.pdf> [Último acceso 1 febrero 2019]

U.S. Geological Survey. 2016. How much water is there on, in, and above the Earth? The USGS Water Science School. [En línea. Acceso libre]. <https://water.usgs.gov/ edu/earthhowmuch.html> [Último acceso 28 febrero 2019] 